\title{
Circular RNA_101237 mediates anoxia/reoxygenation injury by targeting let-7a-5p/IGF2BP3 in cardiomyocytes
}

\author{
JIANTING GAN, JUN YUAN, YU LIU, ZHENGDE LU, YAN XUE, LEI SHI and HUAYUAN ZENG
}

Department of Cardiology, The People's Hospital of Guangxi Zhuang Autonomous Region, Nanning, Guangxi Zhuang Autonomous Region 530021, P.R. China

Received July 3, 2019; Accepted November 20, 2019

DOI: $10.3892 /$ ijmm.2019.4441

\begin{abstract}
Circular RNAs (circRNAs) serve important roles in cardiovascular diseases, including myocardial infarction. However, the mechanisms underlying the roles of circRNAs in cardiomyocyte death induced by anoxia/reoxygenation (A/R) are not fully understood. In the present study, the roles of circRNA_101237 and let-7a-5p in cardiomyocyte death induced by $\mathrm{A} / \mathrm{R}$ injury were investigated. It was identified that circRNA_101237 was induced by A/R injury in a time-dependent manner and that circRNA_101237 knockdown protected cardiomyocytes from A/R-mediated apoptosis. Additional mechanistic studies revealed that circRNA_101237 served as a sponge for let-7a-5p, subsequently regulating insulin-like growth factor $2 \mathrm{mRNA}$-binding protein 3 (IGF2BP3)-dependent autophagy. IGF2BP3 downregulation decreased the levels of apoptosis and inhibited autophagy induced by A/R challenge in primary cardiomyocytes. These results identified circRNA_101237 as a novel circRNA that regulates cardiomyocyte death and autophagy, and demonstrated that the circRNA-101237/let-7a-5p/IGF2BP3 axis, which serves as a regulator of cardiomyocyte death, may be a potential therapeutic target for the management of cardiovascular diseases.
\end{abstract}

\section{Introduction}

Circular RNAs (circRNAs), which are formatted by connecting the $3^{\prime}$ and $5^{\prime}$ ends together via exon circularization or intron circularization, are non-coding RNAs that serve as competing endogenous RNAs $(1,2)$. circRNAs are resistant to RNase R degradation and this stability underlies their abundant expression and evolutionary conservation in human and mice (3). The vast majority of circRNAs are expressed in the cytoplasm and

Correspondence to: Professor Jun Yuan, Department of Cardiology, The People's Hospital of Guangxi Zhuang Autonomous Region, 6 Taoyuan Road, Nanning, Guangxi Zhuang Autonomous Region 530021, P.R. China

E-mail: nnyuanjun@sohu.com

Key words: circular RNA, anoxia/reoxygenation, cardiovascular disease, let-7, insulin-like growth factor 2 mRNA-binding protein 3 contain microRNA (miRNA) response elements to interact with miRNAs and regulate target gene expression during and subsequent to transcription and (4). In addition, circRNAs exhibit tissue-specific expression profiles.

circRNAs serve important roles in cardiovascular diseases, including myocardial infarction, cardiac senescence, atherosclerosis and pathological hypertrophy (5). For instance, heart-associated circRNA (HACR) inhibits miR-223 activity to enhance the protective role of ARC, thereby preventing cardiac hypertrophy and cardiac failure (6). During myocardial infarction, circRNA Cdrlas activates SP1 and PARP by functioning as a sponge for miR-7 (7). Conversely, circ-Foxo3 may inhibit the nuclear translocation of transcription factors and promote cardiac senescence (8). Accordingly, recent studies have suggested that circRNAs may be used as biomarkers and therapeutic targets for cardiovascular diseases $(9,10)$.

circRNAs exert their functions by binding to miRNAs, indicating that circRNAs may serve as upstream regulators of miRNAs (11). Conversely, miRNAs control cardiac function by targeting a number of $\mathrm{mRNA}$ to regulate the proliferation and apoptosis of cells during disease progression (12). In addition, circRNAs are more stable and abundant than miRNAs in serum and other body fluids, and in extracellular vesicles (13). Therefore, circRNAs are ideal candidates as potential disease biomarkers and therapeutic targets (14). However, the mechanisms underlying the roles of circRNAs in cardiomyocyte death induced by anoxia/reoxygenation $(\mathrm{A} / \mathrm{R})$ or ischemia/reperfusion (I/R) injury are not yet fully understood.

The present study investigated the roles of circRNA-101237, encoded by the cyclin dependent kinase 8 (CDK8) gene, and let-7a-5p in cardiomyocyte death induced by A/R injury and the underlying mechanisms. It was demonstrated that circRNA-101237 mediated apoptosis in cardiomyocytes by activating autophagy that was dependent on let-7a-5p/insulin-like growth factor 2 mRNA-binding protein 3 (IGF2BP3) axis. The present study, which identified circRNA_101237 as a novel circRNA, also revealed that the circRNA-101237/let-7a-5p/IGF2BP3 axis may serve as a regulator of cardiomyocyte death and a potential therapeutic target for the management of cardiovascular diseases.

\section{Materials and methods}

Primary cardiomyocyte culture and treatment. Animal experiments were approved by the Ethics Committee for 
Animal Research of The People's Hospital of Guangxi Zhuang Autonomous Region. Cardiomyocytes were isolated from 2-day-old mice as previously described (15). The isolated cardiomyocytes were cultured in DMEM/F-12 (Invitrogen; Thermo Fisher Scientific, Inc.) supplemented with $5 \%$ heat-inactivated horse serum and diluted to $1 \times 10^{6}$ cells $/ \mathrm{ml}$ for $24 \mathrm{~h}$. Subsequently, the cardiomyocytes were passaged in culture dishes coated with laminin. For the A/R treatment model, anoxia was induced by exposure to $5 \% \mathrm{CO}_{2}$ and $95 \% \mathrm{~N}_{2}$ for $2 \mathrm{~h}$, followed by reoxygenation with $95 \% \mathrm{O}_{2}$ and $5 \% \mathrm{CO}_{2}$ for 4,8 or $12 \mathrm{~h}$

circRNA microarray analysis. Cardiomyocytes underwent $\mathrm{A} / \mathrm{R}$ treatment for $12 \mathrm{~h}$ and RNA was extracted using the RNeasy Mini kit (Qiagen China Co., Ltd.) according to the manufacturer's protocol. The total RNA was used to analyze the circRNA expression profiles using the Mouse Circular RNA Array Service (Aksomics Inc.). Differentially expressed circRNAs were identified as fold change $\geq 2$ and $\mathrm{P}<0.001$.

Reverse transcription-quantitative polymerase chain reaction $(R T-q P C R)$. Total RNA was extracted using TRIzol ${ }^{\circledR}$ reagent (Thermo Fisher Scientific, Inc.). Following treatment with DNAse I (Thermo Fisher Scientific, Inc.), RNA was reverse transcribed with reverse transcriptase (Thermo Fisher Scientific, Inc.). RT-qPCR for mature miRNAs was conducted using an All-in-One ${ }^{\mathrm{TM}}$ miRNA RT-qPCR (GeneCopoeia, Inc.) following the manufacturer's recommended protocol on a CFX96 Real-Time PCR Detection System (Bio-Rad Laboratories, Inc.). U6 was used as internal control for miRNA expression analysis. The expression of circRNAs and mRNA was measured by SsoFast ${ }^{\mathrm{TM}}$ EvaGreen $^{\circledR}$ supermix (cat no. 1725201; Bio-Rad Laboratories, Inc.), according to the manufacturer's protocols. Expression of GAPDH was used as an internal control. qPCR was performed with the following thermocycling conditions: $95^{\circ} \mathrm{C}$ for $3 \mathrm{~min}$, and 39 cycles of $95^{\circ} \mathrm{C}$ for $10 \mathrm{sec}$ and $60^{\circ} \mathrm{C}$ for $30 \mathrm{sec}$. The data were analyzed via the comparative quantification cycle method $\left(2^{-\Delta \Delta \mathrm{Cq}}\right.$; $-\Delta \mathrm{Cq}=\mathrm{Cq}_{\text {target gene }}-\mathrm{Cq}_{\text {internal control }}$ ), as previously described (16). The sequences of the circRNAs divergent primers, let-7a-5p, IGF2BP3 and GAPDH primers are listed in Table SI.

Terminal deoxynucleotidyl-transferase-mediated dUTP nick end labelling (TUNEL) assay. Apoptotic cells were analyzed using the TUNEL Chromogenic Apoptosis Detection kit (GeneCopoeia, Inc.) following the manufacturer recommended protocol.

Viral constructions and infection. The circRNA_101237 vector was synthesized by Sangon Biotech Co., Ltd. circRNA_101237 sequence (1 kb upstream) was inserted into pcDNA3.1. circRNA_101237 without the downstream reverse sequence was used as a negative control. All vectors, including circRNA_101237 and IGF2BP3, were finally cloned into the Adeno-X ${ }^{\mathrm{TM}}$ Tet-On Expression System (Seebio Biotech, (Shanghai) Co., Ltd.) according to the manufacturer's protocol. The target sequence of IGF2BP3 small interfering RNA (siRNA) was 5'-AAATGATATTGCTTCCATGAATCTT-3'. The target sequence of circRNA_101237 siRNA was 5'-TCT GATAGTAAGTCTTCG-3'. These siRNA adenoviruses were constructed using the AAVPrime ${ }^{\mathrm{TM}}$ AAV System (GeneCopoeia, Inc.) according to the manufacturer's protocol. All constructs were amplified in GCI-AAV-293Ta cells (GeneCopoeia, Inc.). Primary cardiomyocytes were infected with viral at multiplicity of infection $=50$ for $48 \mathrm{~h}$.

Let-7a-5p mimic and inhibitor transfection. The let-7a-5p mimic and inhibitor, and their negative control were obtained from GeneCopoeia (Guangzhou, China). Let-7a-5p mimic and inhibitor transfection was performed by using Lipofectamine ${ }^{\circledR}$ 3000 (Invitrogen; Thermo Fisher Scientific, Inc.) following the manufacturer's protocol.

Lactate dehydrogenase ( $\mathrm{LDH}$ ) activity measurement. Primary cardiomyocytes were exposed to A/R treatment for $0,4,8$ or $12 \mathrm{~h}$, and the medium was collected for LDH activity measurement using an LDH Quantification kit (cat no. K726-500; BioVision, Inc.) according to the manufacturer's protocol.

Caspase 3 activity measurement. Following transfection, primary cardiomyocytes were exposed to A/R for $8 \mathrm{~h}$, and the cells were collected for caspase 3 activity measurement using a Caspase-3 Fluorometric Assay lit (cat no. K105-100; BioVision, Inc.) according to the manufacturer's protocol.

Autophagic vacuoles staining. Following transfection with circRNA_101237 or scramble control siRNA, primary cardiomyocytes were exposed to $\mathrm{A} / \mathrm{R}$ for $8 \mathrm{~h}$, and the cells were collected for autophagic vacuoles staining using a CYTO-ID autophagy detection kit (cat no. ENZ-51031-0050; Enzo Life Sciences, Inc.) according to the manufacturer's protocol.

Western blot analysis. Following the aforementioned treatments, the cells were lysed by RIPA lysis buffer (Cell Signaling Technology, Inc.) for $30 \mathrm{~min}$ on ice. The BCA Protein Concentration Assay kit (Thermo Fisher Scientific, Inc.) was used to determine the protein concentration of the sample, according to manufacturer's protocol. The $50 \mathrm{~g}$ protein sample were subjected to $10 \%$ SDS-PAGE and transferred to PVDF membranes. The membranes were blocked by $5 \%$ non-fat milk for $1 \mathrm{~h}$ at room temperature. The membranes then were incubated with primary antibodies [anti-microtubule-associated proteins 1A/1B light chain 3B (LC3B), 1:1,000, cat. no. ab48394; anti-IGF2BP3, 1:100, cat. no. ab225697; Beclin1, 1:1,000, cat. no. ab210498; autophagy protein 5 (Atg5), 1:1,000, cat. no. ab228668; and anti-GAPDH, 1:2,000, cat. no. ab8245] overnight at $4^{\circ} \mathrm{C}$. All antibodies were purchased from Abcam. The membranes were washed three times with PBS. Following washing, the membranes were incubated with appropriate secondary antibodies [goat anti-rabbit IgG H\&L (HRP), 1:3,000, cat. no. ab205718; and goat anti-mouse IgG H\&L (HRP), 1:3,000, cat. no. ab205719; both Abcam] for $1 \mathrm{~h}$ at $37^{\circ} \mathrm{C}$. The bands were measured using a chemiluminescence system (Bio-Rad, USA) imaging system.

Luciferase activity assay. PCR was performed to generate the IGF2BP3 3'-untranslated region (UTR) as previously described (17). A QuikChange Lightning Multi Site-Directed Mutagenesis kit (Agilent Technologies, Inc.) was used to generate the mutated 3'-UTR of IGF2BP3. Sequencing 
A

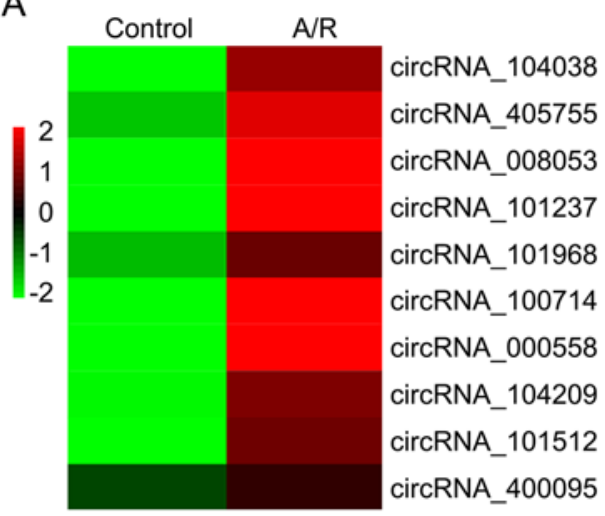

B

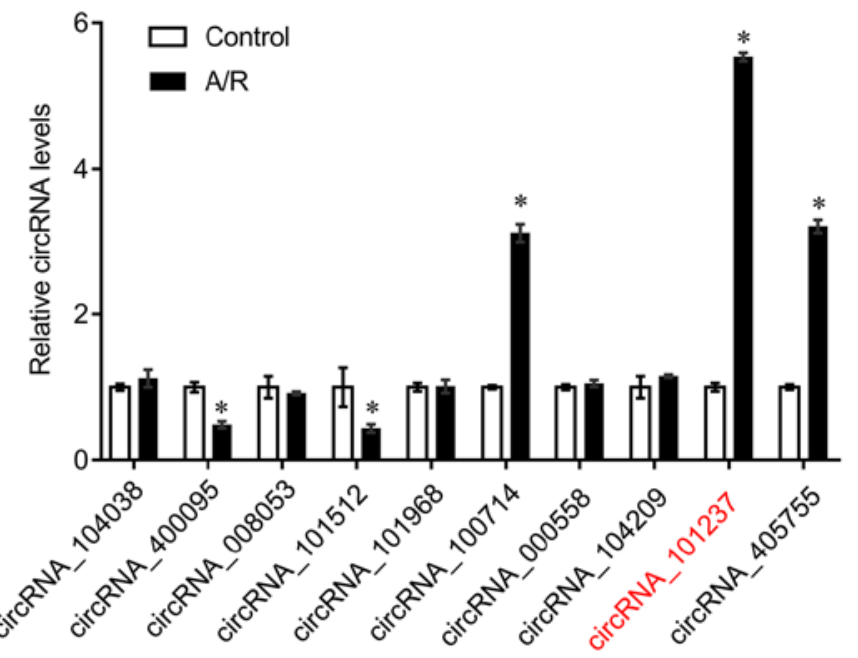

C

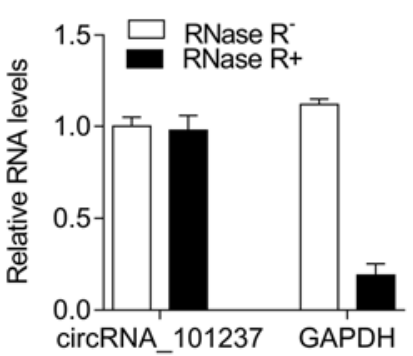

D

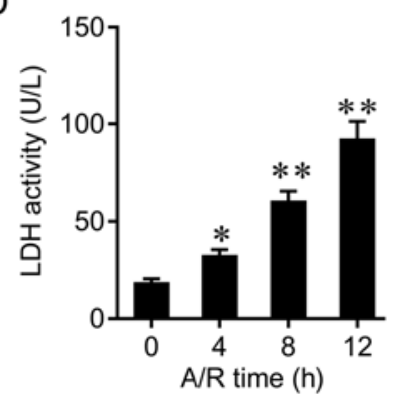

E

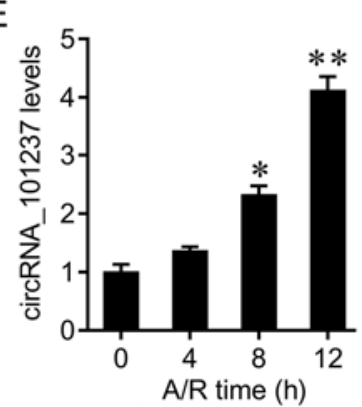

$\mathrm{F}$

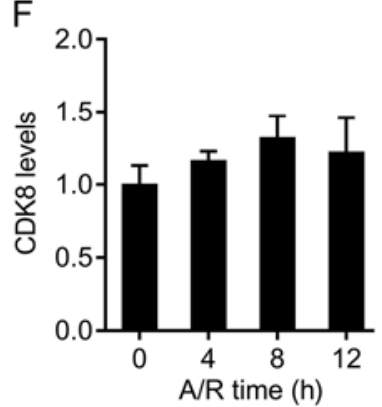

Figure 1. Expression of circRNA in A/R treated cardiomyocytes. (A) A heatmap indicating the differentially expressed circRNA following A/R treatment. (B) RT-qPCR was performed to confirm the circRNAs expression in primary cardiomyocytes following A/R treatment. (C) RT-qPCR was performed to detect circRNA_101237 expression in cardiomyocytes treated with or without RNase R. (D) LDH activity was measured following A/R treatment. (E) circRNA_101237 expression was determined by RT-qPCR following A/R treatment in cardiomyocytes. (F) The expression of the circRNA_101237 host gene, CDK8, was determined by RT-qPCR following A/R treatment in cardiomyocytes. ${ }^{*} \mathrm{P}<0.05$ and ${ }^{* *} \mathrm{P}<0.01$. circRNA, circular RNA; A/R, anoxia/reoxygenation; RT-qPCR, reverse transcription-quantitative polymerase chain reaction; $\mathrm{LDH}$, lactate dehydrogenase; CDK8, cyclin dependent kinase 8 .

was performed to verify coding sequences. Wild-type and mutated 3'-UTRs of IGF2BP3 were subcloned into the pGL3 vector immediately downstream of the luciferase gene (Genomeditech). Primary cardiomyocytes were transfected with wild-type or mutated 3'-UTRs of IGF2BP3, and co-transfected with let-7a-5p mimics or mimics negative control using Lipofectamine ${ }^{\circledR} 3000$ (Thermo Fisher Scientific, Inc.). The cells transfected with pGL3 vector were used as control. At $48 \mathrm{~h}$ after transfection, the Dual-Luciferase Reporter Assay System (Promega Corporation) was used to determine the luciferase activity according to the manufacturer's protocol. Renilla luciferase activity was used for normalization.

Biotinylated miRNA pull-down. The assay was performed as previously described (1). The 5'-biotin labeled let-7a-5p was synthesized by Sangon Biotech Co., Ltd. Cardiomyocytes were transfected with biotinylated let-7a-5p for $24 \mathrm{~h}$. Following transfection, cardiomyocytes were lysed in RIPA lysis buffer (Cell Signaling Technology, Inc.) for $30 \mathrm{~min}$ on ice and centrifuged at $12,000 \mathrm{x} \mathrm{g}$ at $4^{\circ} \mathrm{C}$ for $20 \mathrm{~min}$ to collect the supernatant. A sample of $50 \mu \mathrm{l}$ was used as an input control. The supernatant was incubated with streptavidin agarose beads (Invitrogen; Thermo Fisher Scientific, Inc.) at $4^{\circ} \mathrm{C}$ for $3 \mathrm{~h}$. The bound RNAs were purified by washing with low-salt buffer and high-salt buffer (Wuhan Boster Biological Technology, Ltd.). The purified RNA was used for RT-qPCR according to the aforementioned protocol. GAPDH was used as a negative control.

Protein argonaute 2 (AGO2) immunoprecipitation. Let-7a-5p and let-7a-5p mutants were transfected into cardiomyocytes for $48 \mathrm{~h}$. The cells were lysed in RIPA lysis buffer (Cell Signaling Technology, Inc.) for $30 \mathrm{~min}$ on ice and incubated with anti-AGO2 (1:100; cat. no. ab186733; Abcam) or control IgG antibody-(1:100; cat. no. 3900s; Cell Signaling Technology, Inc.) coupled Sepharose beads for $4 \mathrm{~h}$ at $4^{\circ} \mathrm{C}$ under rotation. The bound RNAs were purified by washing with lysis buffer (Wuhan Boster Biological Technology, Ltd.). The purified RNA was used for RT-qPCR according to the aforementioned protocol. GAPDH was used as a negative control.

Immunofluorescence assays. The cardiomyocytes were cultured on slides for $24 \mathrm{~h}$. Following blocking with non-fat $5 \%$ milk for $60 \mathrm{~min}$ at $37^{\circ} \mathrm{C}$, the slides were incubated with an anti-LC3B primary antibody (1:200 dilution; cat. no. ab48394; Abcam) at $4^{\circ} \mathrm{C}$ overnight. The slides were 

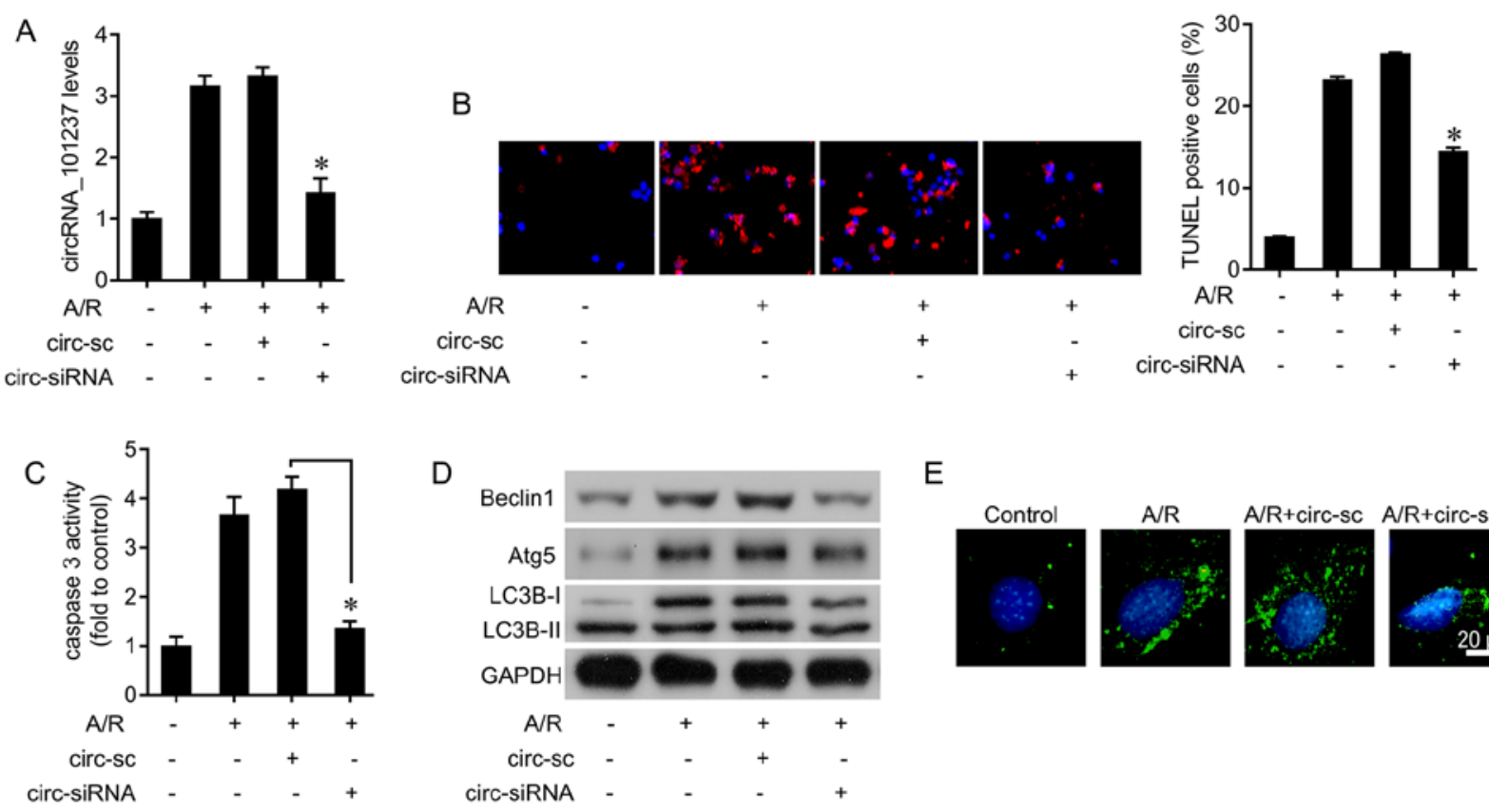

E
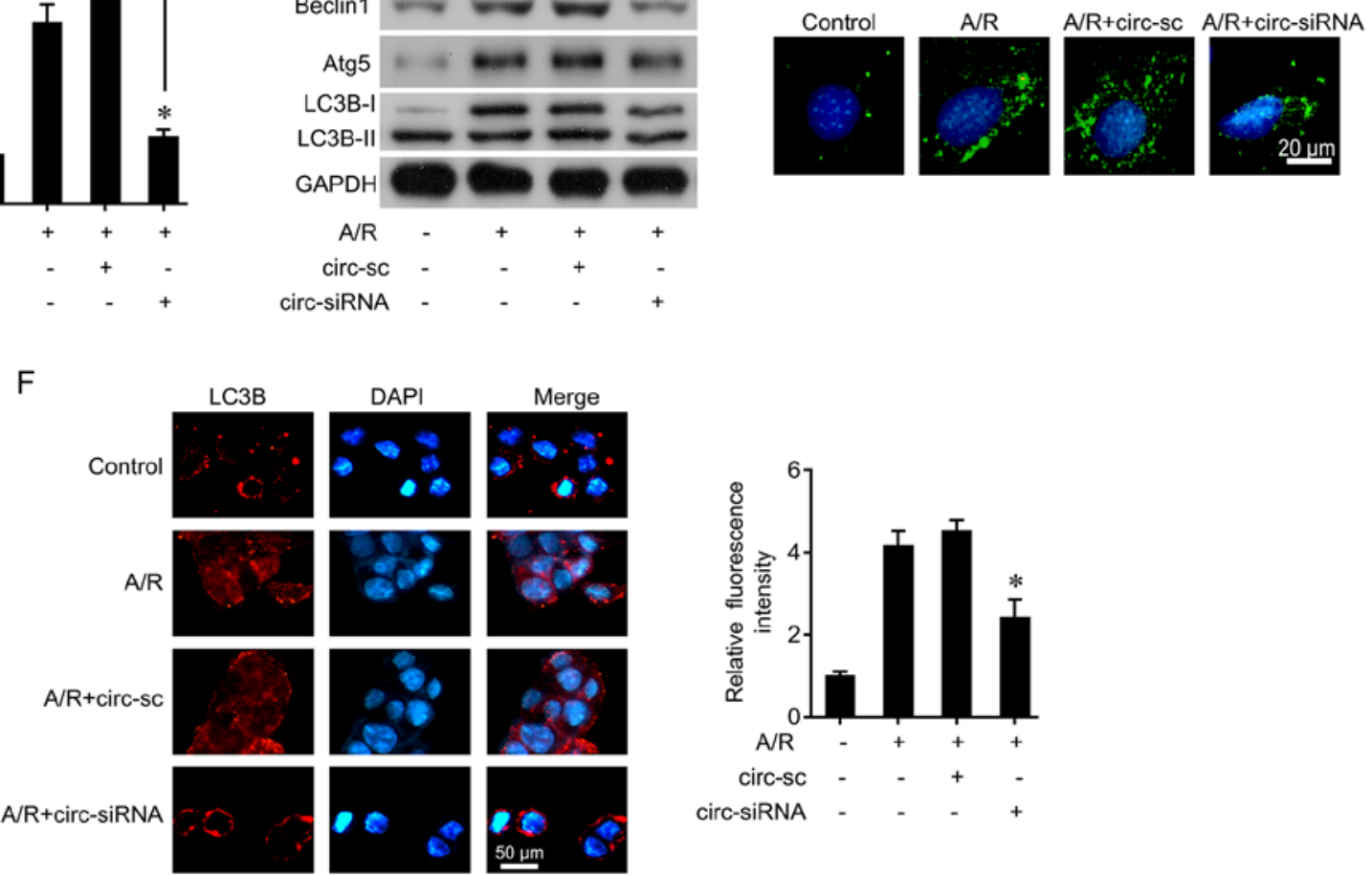

Figure 2. circRNA_101237 knockdown inhibits apoptosis in cardiomyocytes. (A) circRNA_101237 levels were analyzed by RT-qPCR following the indicated treatment in cardiomyocytes. (B) TUNEL staining was performed to determine the apoptotic cells following circRNA_101237 knockdown and A/R treatment in cardiomyocytes. (C) Caspase 3 activity was determined after circRNA_101237 knockdown and A/R treatment in cardiomyocytes. (D) The autophagy markers Beclin1, Atg5 and LC3B were analyzed by western blot analysis following circRNA_101237 knockdown and A/R treatment in cardiomyocytes. (E) Autophagic vacuoles were staining with Cyto-ID Green dye following circRNA_101237 knockdown and A/R treatment in cardiomyocytes. (F) Levels of the autophagy marker LC3B were analyzed by immunofluorescence following circRNA_101237 knockdown and A/R treatment in cardiomyocytes. " $\mathrm{P}<0.05$ vs. A/R alone. circRNA, circular RNA; A/R, anoxia/reoxygenation; RT-qPCR, reverse transcription-quantitative polymerase chain reaction; TUNEL, terminal deoxynucleotidyl-transferase-mediated dUTP nick end labelling; Atg5, autophagy protein 5; LC3B, microtubule-associated proteins 1A/1B light chain 3B.

incubated with appropriate secondary antibodies for $1 \mathrm{~h}$ at $37^{\circ} \mathrm{C}$. Following washing with PBS, the slides were co-stained with DAPI $(1: 2,000$; cat. no. SC-3598; Santa Cruz Biotechnology, Inc.) for 2 min at room temperature. Immunofluorescence images were captured at x200 magnification using a fluorescence microscope (Nikon ECLIPSE 80i; Nikon Corporation).

Bioinformatics analysis. The bioinformatics software RNAhybrid (version 2.1; https://bibiserv.cebitec.uni-bielefeld. de/rnahybrid/)was used to identify potential miRNA binding sites within circRNA_101237, as previously described (18). The bioinformatics tool miRwalk (version 2; http://zmf.umm. uni-heidelberg.de/apps/zmf/mirwalk2/) was used to predict the potential targets of let-7a-5p as previously described (19).
Statistical analysis. All statistical analyses were performed using GraphPad Prism v.7.04 (GraphPad Software, Inc.). Continuity variables are expressed as mean \pm standard deviation of at least 3 independent experiments. A Student's t-test was used for comparison between two groups; a one-way analysis of variance was used for comparisons between three or more groups, followed by a Tukey's post hoc test for comparison. All statistical analyses were performed using a two-sided test. $\mathrm{P}<0.05$ was considered to indicate a statistically significant difference.

\section{Results}

circRNA_101237 is induced by $A / R$ in cardiomyocytes. To investigate the potential role of circRNAs A/R-induced 
A

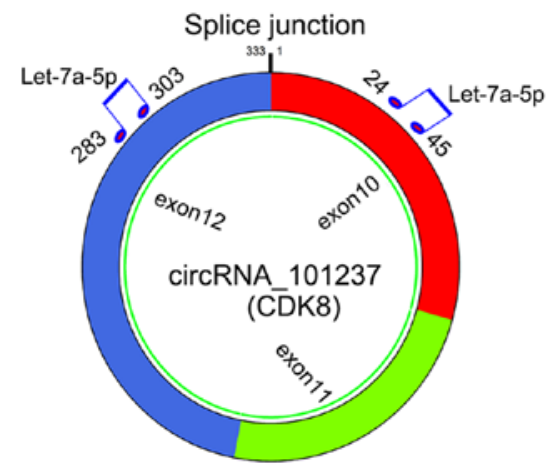

Bio-Let-7a-5p wt

3' -UUGAUAUGUUGGAUGAUGGAGU-5'

Bio-Let-7a-5p mut

3' -UUGAUAUGUUGGAUGCCAAGAC-5

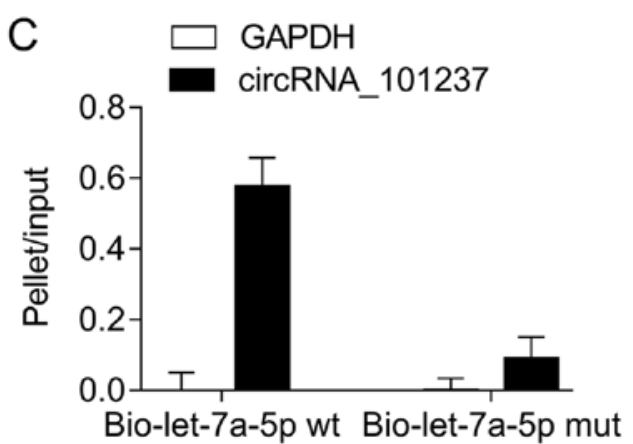

B
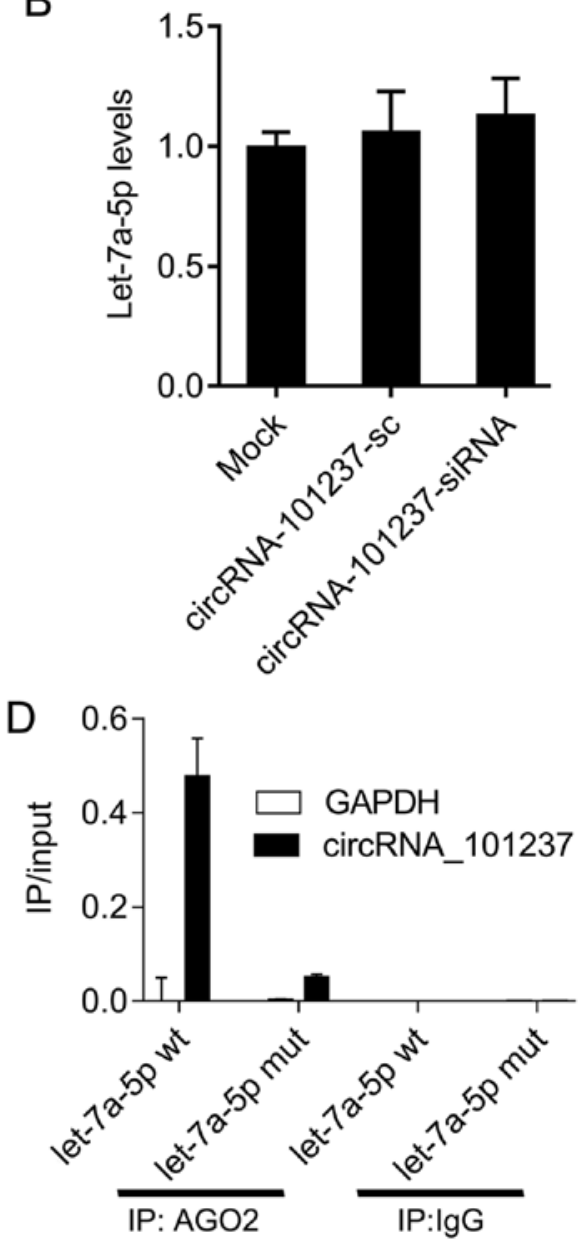

Figure 3. circRNA_101237 serves as a let-7a-5p sponge. (A) circRNA_101237 contains two sites complementary to let-7a-5p, as identified by the bioinformatics program RNAhybrid (upper panel). The biotin-coupled let-7a-5p mutant is presented in the lower panel. (B) RT-qPCR was performed to determine let-7a-5p levels following circRNA_101237 knockdown. (C) IP was performed to analyze the interaction between let-7a-5p and circRNA_101237. (D) IP of AGO2 from cardiomyocytes transfected with let-7a-5p or let-7a-5p mutant. circRNA_101237 and GAPDH mRNA levels were quantified by RT-qPCR, and the relative IP/input ratios were plotted. circRNA, circular RNA; RT-qPCR, reverse transcription-quantitative polymerase chain reaction; IP, immunoprecipitation; AGO2, protein argonaute 2 .

cardiomyocyte death, a circRNA microarray was performed to screen the differentially expressed circRNAs in A/R-treated cardiomyocytes. The heatmap demonstrates the 10 circRNAs that were differentially expressed following A/R treatment (Fig. 1A). Their expression levels were confirmed by RT-qPCR. The results indicated that circRNA_400095 and circRNA_101512 levels were decreased, and circRNA_100714, circRNA_405755 and circRNA_101237 levels were increased upon A/R treatment. circRNA_101237 levels, generated from the exon 10 to exon 12 of the CDK8 gene, were significantly increased by A/R stimulation (Fig. 1B). Due to resistance to RNase R treatment, circRNAs cannot be degraded by RNase R digestion. It was identified that GAPDH mRNA (linear RNA transcripts) was degraded by RNase while circRNA_101237 was not degraded (Fig. 1C). Primary cardiomyocytes were then subjected to $\mathrm{A} / \mathrm{R}$ for different times. $\mathrm{LDH}$ is an enzyme located in the cytoplasm. When the cell membrane is damaged by stimuli, such as A/R challenges, LDH is released. The levels of $\mathrm{LDH}$ released may reflect the cellular response to A/R (20). Therefore, $\mathrm{LDH}$ activity was used as a positive control to evaluate whether the A/R was successfully established. Primary cardiomyocytes were exposed to
A/R for $0,4,8$, or $12 \mathrm{~h}$, and the medium was collected for LDH activity measurement using an LDH Quantification kit. LDH activity was significantly upregulated by A/R treatment (Fig. 1D). In addition, circRNA_101237 levels increased in a time-dependent manner, while the expression levels of host gene CDK8 were not significantly different among the groups (Fig. 1E and F). These data suggest that circRNA_101237 may contribute to oxygen deprivation-induced cardiomyocyte death.

Knockdown of circRNA_101237 decreases cardiomyocyte apoptosis. The role of circRNA_101237 in A/R-induced cardiomyocyte apoptosis was next examined by siRNA transfection. circRNA_101237 knockdown efficiently inhibited the increase in circRNA_101237 level induced by A/R in cardiomyocytes (Fig. 2A). A TUNEL assay was used to determine the cell apoptosis induced by A/R. A/R treatment significantly induced cell apoptosis. However, knockdown of circRNA_101237 decreased the level of A/R-induced apoptosis, identified by a decrease in the number of TUNEL-positive cells (Fig. 2B). In addition, knockdown of circRNA_101237 inhibited A/R-induced caspase 3 activity (Fig. 2C). 
A
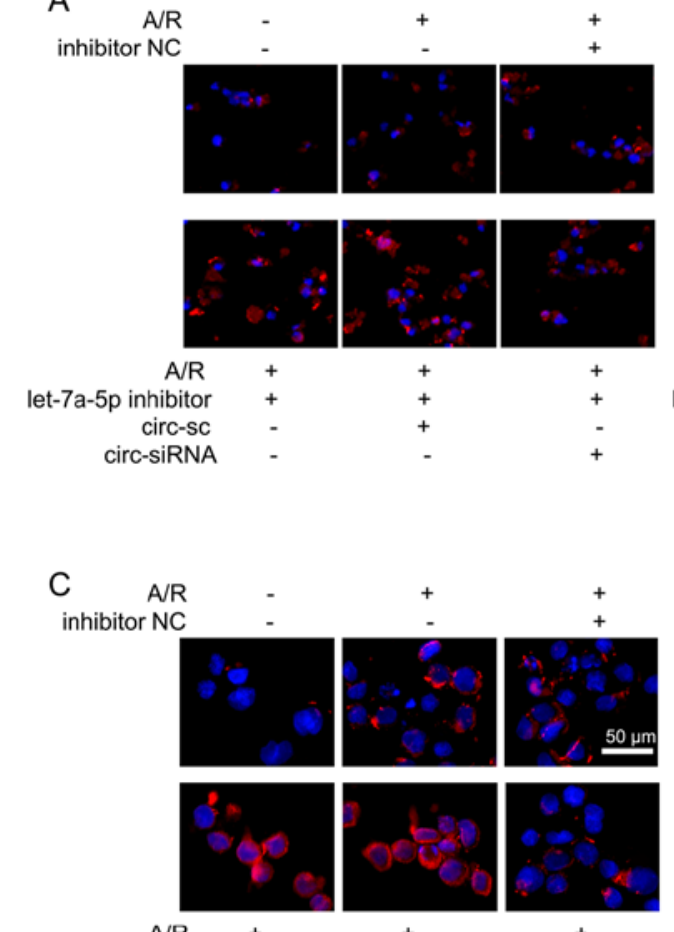

let-7a-5p inhibitor

re-siRNA
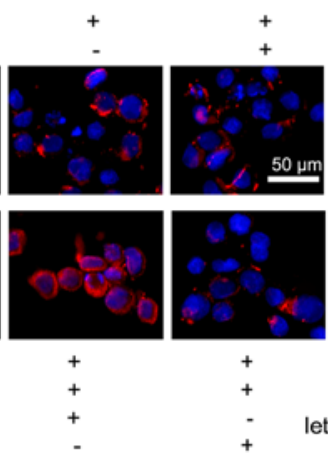

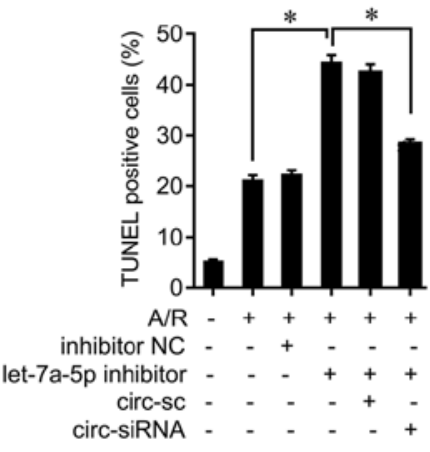

B

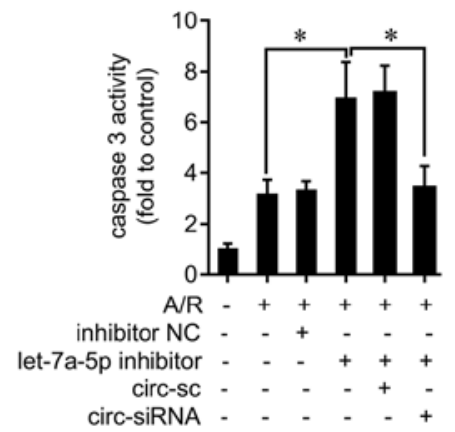

D
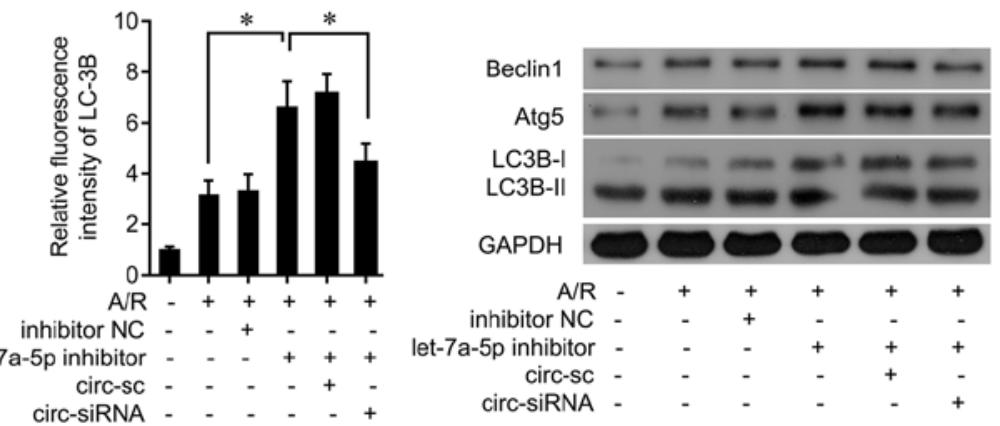

Figure 4. let-7a-5p inhibitor reverses circRNA_101237 knockdown-mediated cardiomyocytes apoptosis. (A) TUNEL staining was performed to determine the levels of apoptotic cells following circRNA_101237 knockdown and let-7a-5p inhibitor treatment in cardiomyocytes. (B) Caspase 3 activity was determined following circRNA_101237 knockdown and let-7a-5p inhibitor treatment in cardiomyocytes. (C) The autophagy marker, LC3B, was analyzed by immunofluorescence following circRNA_101237 knockdown and let-7a-5p inhibitor treatment in cardiomyocytes. (D) The autophagy markers Beclin1, Atg5 and LC3B was analyzed by western blot analysis following circRNA_101237 knockdown and let-7a-5p inhibitor treatment in cardiomyocytes. ${ }^{*} \mathrm{P}<0.05$. circRNA, circular RNA: TUNEL, terminal deoxynucleotidyl-transferase-mediated dUTP nick end labelling; LC3B, microtubule-associated proteins 1A/1B light chain 3B; Atg5, autophagy protein 5 .

Autophagic cell death serves important roles in cardiac diseases, including ischemic heart disease and heart failure (21). The autophagy levels in cells were evaluated following $A / R$ treatment and circRNA_101237 siRNA transfection. It was observed that the expression levels of Beclin1, Atg5, and LC3B were increased by A/R treatment. circRNA_101237 siRNA transfection attenuated A/R-mediated autophagy activation (Fig. 2D). Cyto-ID Green dye was used to staining autophagic vacuoles, and it was identified that $A / R$ treatment significantly increased the number of autophagic vacuoles, which was decreased by circRNA_101237 siRNA transfection (Fig. 2E). In addition, the immunofluorescence assay results also confirmed that circRNA_101237 knockdown reversed A/R-mediated LC3B upregulation (Fig. 2F). These data indicate that circRNA_101237 knockdown inhibits cardiomyocytes apoptosis via the autophagy pathway.

circRNA_101237 serves as a sponge for let-7a-5p. circRNA_101237 is an exonic circRNA. The bioinformatics software RNAhybrid was used to identify potential miRNA binding sites within circRNA_101237. circRNA_101237 interacted with let-7a-5p at the two predicted binding sites (Fig. 3A). However, it was identified that circRNA_101237 knockdown had no effect on let-7a-5p expression (Fig. 3B). The RNA pull-down assays results demonstrated a higher enrichment of circRNA_101237 in the bio-let-7a-5p-captured fraction compared with the negative control (Fig. 3C). Furthermore, AGO2 immunoprecipitation also suggested that endogenous circRNA_101237 and let-7a-5p were co-located with AGO2, but circRNA_101237 was not detectable in samples transfected with mutant let-7a-5p mutant (Fig. 3D). These results suggested that let-7a-5p facilitates the AGO2 association with circRNA_101237. Therefore, circRNA_101237 functions as a let-7a-5p sponge without regulating let-7a-5p expression.

Let-7a-5p inhibitor attenuates the effects of circRNA_101237 on cell apoptosis and autophagy in cardiomyocytes. Next, whether endogenous let-7a-5p was implicated in the cell death induced by $\mathrm{A} / \mathrm{R}$ was explored. Let-7a-5p inhibitors were transfected into primary cardiomyocytes (Fig. S1A). Let-7a-5p inhibition enhanced A/R-induced apoptosis, while co-treatment of circRNA_101237 siRNA attenuated apoptosis (Fig. 4A). In addition, the let-7a-5p inhibitor upregulated A/R-induced caspase 3 activity, which was then inhibited by circRNA_101237 siRNA transfection (Fig. 4B). Furthermore, the let-7a-5p inhibitor significantly increased A/R-induced LC3B levels, which was abolished by circRNA_101237 knockdown (Fig. 4C). The expression levels of key molecules in the autophagy pathway were also examined, and it was 
A

$\begin{array}{lr}\text { let-7a-5p } & \text { 3'-UGAUAUGUUGGAUGAUGGAGU-5' } \\ \text { Mm } & \text { 5'-UAAACGUGCAUUUUU-ACUCAACUACCUCAGGUAUU-3' } \\ \text { Hs } & \text { 5'-UAAACGUGCAUUUUU-ACUCAACUACCUCAGGUAUU-3' } \\ \text { Rn } & \text { 5'-UCAGCGUGCAUUUUUUACUCAACUACCUCAGGUAUU-3' } \\ \text { Cf } & \text { 5'-UAAAUGUGCAUUUUU-ACUCAACUACCUCAGGUAUU-3' } \\ \text { Gg } & \text { 5'-UAAAUAUGCAUUUU--ACUAAACUACCUCAGGCAUU-3' } \\ & \text { Position 547-555 of IGF2BP3 3' UTR }\end{array}$

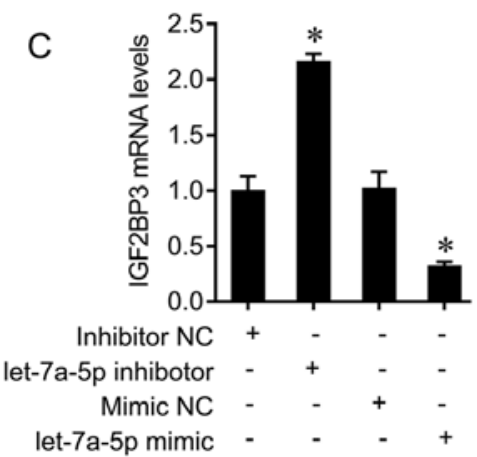

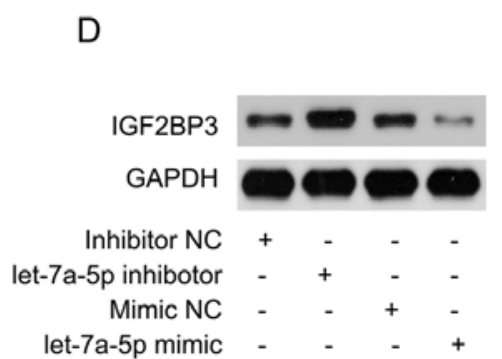

B

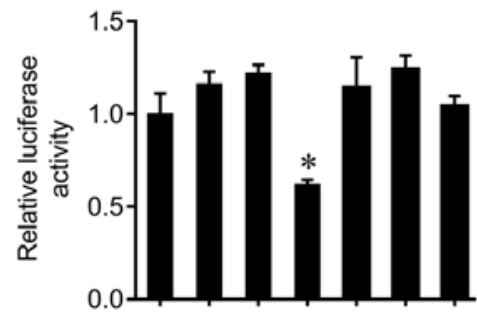

pGL3-Control + - - - - - -

$\begin{array}{rccccccc}\text { IGF2BP3 3'UTR wt } & - & + & + & + & - & - & - \\ \text { IGF2BP3 3'UTR mut } & - & - & - & - & + & + & + \\ \text { Mimic NC } & - & - & + & - & - & + & - \\ \text { let-7a-5p mimic } & - & - & - & + & - & - & +\end{array}$

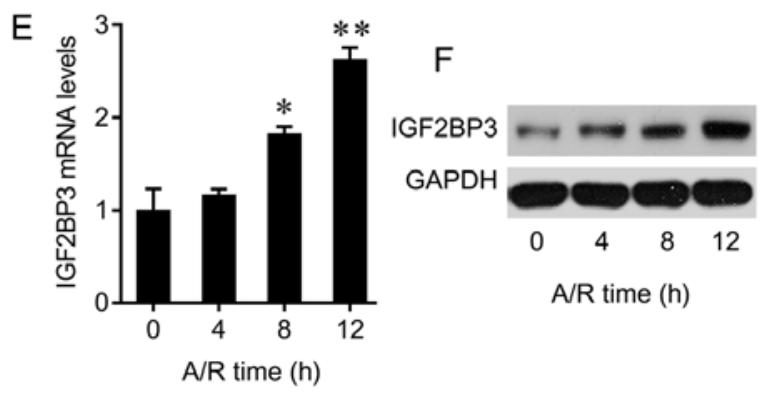

Figure 5. let-7a-5p targets IGF2BP3. (A) The conserved binding site of let-7a-5p on the 3' UTR of IGF2BP3. (B) Wild-type IGF2BP3 3' UTR or mutant 3' UTR and let-7a-5p mimics were transfected into cardiomyocytes. Then, a dual-luciferase assay was performed. ${ }^{*} \mathrm{P}<0.05$ vs. Mimic-NC. (C and D) Cardiomyocytes were transfected with let-7a-5p mimics or let-7a-5p inhibitor. The mRNA and protein expression of IGF2BP3 was analyzed by (C) RT-qPCR and (D) western blot analysis, respectively. "P<0.05 vs. NC. (E and F) Cardiomyocytes were exposed to A/R for different time periods. The expression of IGF2BP3 was analyzed by (E) RT-qPCR and (F) western blot analysis. "P<0.05 and ${ }^{* *} \mathrm{P}<0.01$ vs. 0 h. IGF2BP3, insulin-like growth factor 2 mRNA-binding protein 3; UTR, untranslated region; NC, negative control; $\mathrm{A} / \mathrm{R}$, anoxia/reoxygenation.

identified that treatment with the let-7a-5p inhibitor increased Beclin1, Atg5, and LC3B levels following A/R treatment (Fig. 4D). These data demonstrate that circRNA_101237 serves as a let-7a-5p sponge to promote cardiomyocyte apoptosis through activating autophagy.

Let-7a-5p inhibits cardiomyocytes death by targeting $I G F 2 B P 3$. The bioinformatics tool miRwalk (http://zmf.umm. uni-heidelberg.de/apps/zmf/mirwalk2/) was used to predict the potential targets of let-7a-5p. The 3' UTR of the IGF2BP3 gene harbors the conserved binding sites of let-7a-5p (Fig. 5A). The luciferase assay system was used to confirm the interaction of let-7a-5p and IGF2BP3. Let-7a-5p transfection led to a significant decrease in luciferase activity in primary cardiomyocytes transfected with the wild-type 3'UTR of IGF2BP3; this effect was abolished by mutation of the IGF2BP3 gene (Fig. 5B). In addition, let-7a-5p inhibitor treatment increased the mRNA and protein levels of IGF2BP3, and let-7a-5p mimic transfection increased let-7a-5p levels (Fig. S1A) and decreased IGF2BP3 expression in primary cardiomyocytes (Fig. 5C and D). Therefore, let-7a-5p may interact with IGF2BP3 and suppress IGF2BP3 expression.

It was also observed that $\mathrm{A} / \mathrm{R}$ exposure increased the mRNA and protein levels of IGF2BP3 in cardiomyocytes in a time-dependent manner (Fig. 5E and F). Furthermore, the effect of IGF2BP3 on the cell death induced by A/R treatment was investigated. IGF2BP3 expression was knocked down by siRNA transfection (Fig. S1B and C). IGF2BP3 silencing decreased the number of TUNEL-positive cells induced by $\mathrm{A} / \mathrm{R}$ challenge, while co-treatment with let-7a-5p inhibitors significantly increased the number of TUNEL-positive cells (Fig. 6B). In addition, IGF2BP3 knockdown inhibited caspase 3 activity induced by $A / R$ challenge, which was increased by co-treatment with let-7a-5p inhibitors (Fig. 6B).

The results of the immunofluorescence assay revealed that knockdown of IGF2BP3 inhibited A/R-mediated LC3B upregulation, which was abolished by let-7a-5p inhibitor in primary cardiomyocytes under A/R treatment (Fig. 6C). IGF2BP3 downregulation decreased Beclin1, Atg5, and LC3B levels following A/R treatment (Fig. 6D). The effects of IGF2BP3 downregulation on Beclin1, Atg5, and LC3B levels was attenuated by let-7a-5p inhibitor treatment (Fig. 6D). Therefore, let-7a-5p inhibited cardiomyocyte death by targeting IGF2BP3 and inactivating autophagy.

\section{Discussion}

In cardiovascular diseases, several miRNAs have been suggested as potential biomarkers with high diagnostic and prognostic potential that were comparable to those of established protein-based biomarkers, such as cardiac troponins and natriuretic peptides $(22,23)$. Additionally, miRNAs may also be utilized as therapeutic agents in cardiovascular diseases (24). Recent studies have revealed that circRNAs may function as endogenous miRNA sponges, ultimately exerting a significant effect on downstream miRNA targets. For example, the circRNA MFACR directly interacts with miR-652-3p and inhibits its expression, subsequently inducing mitochondrial 
A
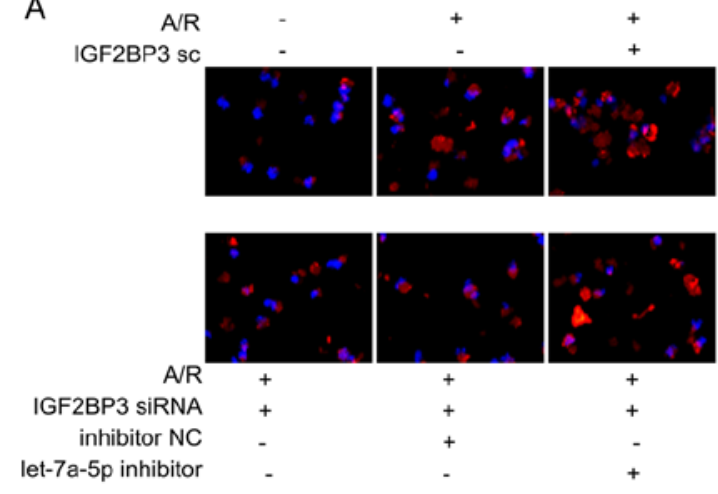

let-7a-5p inhibitor

C
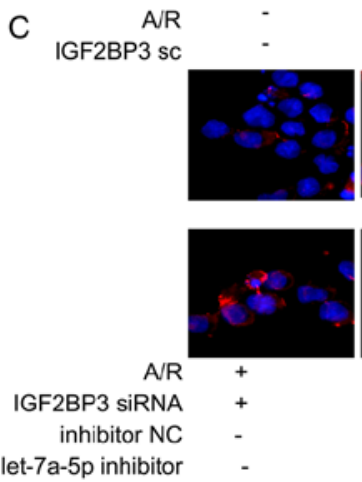
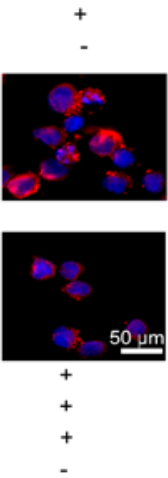
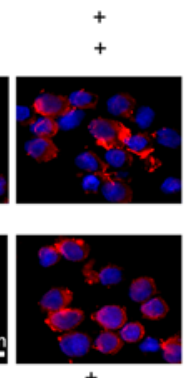

$+$ let-7a-5p inhibitor

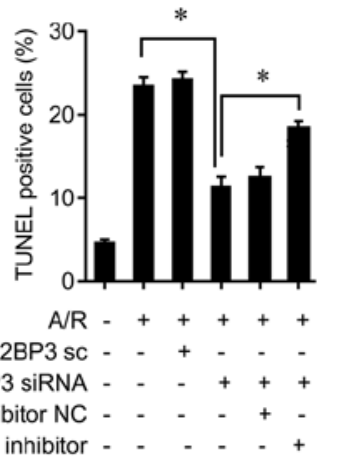
let-7a-5p inhibitor - $\quad-\quad+\quad-+$
B

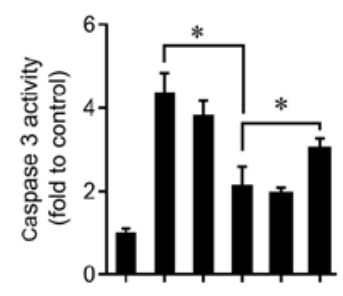

$\mathrm{A} / \mathrm{R}-+++++$ IGF2BP3 SC - - + - - IGF2BP3 SIRNA - - - + + + inhibitor NC - - - - + let-7a-5p inhibitor - - - - + +

Figure 6. let-7a-5p inhibits cardiomyocytes apoptosis by targeting IGF2BP3. (A) Cardiomyocytes were transfected with IGF2BP3 siRNA or control oligo, or let-7a-5p inhibitor, and then exposed to A/R for $12 \mathrm{~h}$. A TUNEL assay was performed to measure levels of cell apoptosis. "P<0.05. (B) Caspase 3 activity was determined following IGF2BP3 knockdown and let-7a-5p inhibitor treatment in cardiomyocytes. (C) Cardiomyocytes were transfected with IGF2BP3 siRNA or control oligo, or let-7a-5p inhibitor, and then exposed to A/R for $12 \mathrm{~h}$. The autophagy marker, LC3B, was analyzed by immunofluorescence. (D) The autophagy markers Beclin1, Atg5 and LC3B was analyzed by western blot analysis following IGF2BP3 knockdown and let-7a-5p inhibitor treatment in cardiomyocytes. "P $<0.05$. IGF2BP3, insulin-like growth factor 2 mRNA-binding protein 3; siRNA, small interfering RNA; TUNEL, terminal deoxynucleotidyl-transferase-mediated dUTP nick end labelling; A/R, anoxia/reoxygenation; LC3B, microtubule-associated proteins 1A/1B light chain 3B; Atg5, autophagy protein 5; $\mathrm{NC}$, negative control.

protein, $18 \mathrm{kDa}$ translation to regulate mitochondrial fission and apoptosis (25), and circRNA HACR serves as an endogenous miR-223 sponge to inhibit cardiac hypertrophy and heart failure by targeting activity-regulated cytoskeleton-associated protein (26). In the present study, a novel and crucial circRNA, circRNA_101237, which regulated cardiomyocyte apoptosis during $A / R$ injury, was identified. It was also demonstrated that circRNA_101237 targeted the let-7a-5p/IGF2BP3 signaling axis, which provided additional evidence for the sponge activity of this circRNA (27).

The sponging of let-7a-5p leads to the upregulation of IGF2BP3 protein and the activation of autophagy. The let-7 family contains 13 members, including let-7a-1 (let-7a-5p), let-7c, let-7d, let-7e, let-7f, let-7g, let-7i, mir-98 and mir-202 (28). Let-7 has been identified as a tumor suppressor in a number of types of human cancer (29). Additionally, the members of let-7 family have been identified to serve important roles in cardiovascular biology and disease (30). Let-7 dysfunction has been observed in various cardiovascular diseases. For example, a previous study demonstrated the upregulation of let-7b and let-7c in cardiac hypertrophy, and the downregulation of let-7a in angiotensin II-induced cardiac hypertrophy (31). However, let-7 inhibition has also been suggested to prevent deterioration of cardiac function via an increase in the recruitment of epicardial cells and EMT (32).
It is important to note that the expression of the let-7 members is disease- and tissue-specific (30). Let-7a is upregulated during cardiac development and downregulated in human endothelial cells during angiogenesis $(33,34)$, indicating that the changes in the expression of let-7 family members are specific to certain pathological processes, and highlights the requirement for an improved understanding of the let-7 family function. In the present study, the results revealed that let-7a-5p expression was unchanged by circRNA_101237 knockdown. However, it was also demonstrated that let-7a-5p inhibition lead to the augmentation of A/R-mediated cardiomyocyte death. Further examination identified IGF2BP3 as a novel let-7a-5p target. IGF2BP3 is a oncofetal protein that refines the post-transcriptional modulation of gene expression (35). IGF2BP3 can bind to the 5' UTR of IGF2 and regulate IGF2 expression and function (36). IGF2 activates the PI3K/AKT pathway, which is involved in autophagy and apoptosis (37). Autophagy is an essential cellular process in the heart and impaired autophagy serves a causative role in cardiomyopathy. Stimuli such as A/R and I/R injury may lead to excessive autophagic activity, which can damage a large fraction of the cytoplasm and organelles, in particular the mitochondria, subsequently leading to cell death (38). Therefore, maintaining cellular autophagic homeostasis is important for cardiomyocyte survival. 
In the present study, it was identified that IGF2BP3 was involved in A/R-induced cardiomyocyte apoptosis and, to the best of our knowledge, demonstrated for the first time that IGF2BP3 was a target of let-7a-5p. It was also demonstrated that circRNA_101237 served as a sponge for let-7a-5p and participated in the regulation of autophagy and cardiomyocyte death in response to $\mathrm{A} / \mathrm{R}$ challenge. Cardiomyocyte death as a result of low oxygen levels is regulated by complex signaling pathways (39). Although the present study indicated that circRNA_101237 may regulate cardiomyocyte apoptosis and autophagy induced by A/R injury, the mechanism underlying the generation of circRNA_101237 and the molecular mechanisms underlying the upregulation of circRNA_101237 in cardiomyocytes remain unknown. As miRNAs can contain several circRNA binding sites, it remains a possibility that other circRNAs in addition to circRNA-101237 may also control the expression of let-7a-5p.

In conclusion, to the best of our knowledge, this was the first study to demonstrate the regulatory mechanism and the roles of circRNA_101237 in A/R injury. The results identified a novel circRNA_101237/let-7a-5p/IGF2BP3 axis, which regulated cardiomyocyte apoptosis via autophagy regulation, as a potential therapeutic target for the management of cardiovascular diseases.

\section{Acknowledgements}

Not applicable.

\section{Funding}

No funding was received.

\section{Availability of data and materials}

All data generated or analyzed during this study are included in this published article.

\section{Authors' contributions}

JG and HZ designed the study. JY and LS performed the cell biological experiments. YL, ZL, and YX performed the reverse transcription-quantitative polymerase chain reaction, western blot, luciferase reporter assay and immunofluorescence staining assays. All authors contributed to the writing of the manuscript. All authors read and approved the final manuscript.

\section{Ethics approval and consent to participate}

The present study was approved by the Ethics Committee of The People's Hospital of Guangxi Zhuang Autonomous Region.

\section{Patient consent for publication}

Not applicable.

\section{Competing interests}

The authors declare that they have no competing interests.

\section{References}

1. Wang K, Long B, Liu F, Wang JX, Liu CY, Zhao B, Zhou LY, Sun T, Wang M, Yu T, et al: A circular RNA protects the heart from pathological hypertrophy and heart failure by targeting miR-223. Eur Heart J 37: 2602-2611, 2016.

2. Jakobi T, Czaja-Hasse LF, Reinhardt $R$ and Dieterich $C$ : Profiling and validation of the circular RNA repertoire in adult murine hearts. Genomics Proteomics Bioinformatics 14: 216-223, 2016.

3. Xu S, Zhou L, Ponnusamy M, Zhang L, Dong Y, Zhang Y, Wang Q, Liu J and Wang K: A comprehensive review of circRNA: From purification and identification to disease marker potential. PeerJ 6: e5503, 2018.

4. Li JJ, Wang W, Wang XQ, He Y, Wang SS and Yan YX: A novel strategy of identifying circRNA biomarkers in cardiovascular disease by meta-analysis. J Cell Physiol 234: 21601-21612, 2019.

5. Fan X, Weng X, Zhao Y, Chen W, Gan T and Xu D: Circular RNAs in cardiovascular Disease: An overview. Biomed Res Int 2017: 5135781, 2017.

6. Burd CE, Jeck WR, Liu Y, Sanoff HK, Wang Z and Sharpless NE: Expression of linear and novel circular forms of an INK4/ARF-associated non-coding RNA correlates with atherosclerosis risk. PLoS Genet 6: e1001233, 2010.

7. Memczak S, Jens M, Elefsinioti A, Torti F, Krueger J, Rybak A, Maier L, Mackowiak SD, Gregersen LH, Munschauer M, et al: Circular RNAs are a large class of animal RNAs with regulatory potency. Nature 495: 333-338, 2013.

8. Du WW, Yang W, Chen Y, Wu ZK, Foster FS, Yang Z, Li X and Yang BB: Foxo3 circular RNA promotes cardiac senescence by modulating multiple factors associated with stress and senescence responses. Eur Heart J 38: 1402-1412, 2017.

9. Zhou Q, Zhang Z, Bei Y, Li G and Wang T: Circular RNAs as novel biomarkers for cardiovascular diseases. Adv Exp Med Biol 1087: 159-170, 2018.

10. Wang L, Meng X, Li G, Zhou Q and Xiao J: Circular RNAs in cardiovascular diseases. Adv Exp Med Biol 1087: 191-204, 2018.

11. Tan WL, Lim BT, Anene-Nzelu CG, Ackers-Johnson M, Dashi A, See K, Tiang Z, Lee DP, Chua WW, Luu TD, et al: A landscape of circular RNA expression in the human heart. Cardiovasc Res 113: 298-309, 2017.

12. Altesha MA, Ni T, Khan A, Liu K and Zheng X: Circular RNA in cardiovascular disease. J Cell Physiol 234: 5588-5600, 2019.

13. Kristensen LS, Andersen MS, Stagsted L, Ebbesen KK, Hansen TB and Kjems J: The biogenesis, biology and characterization of circular RNAs. Nat Rev Genet 20: 675-691, 2019.

14. Bayoumi AS, Aonuma T, Teoh JP, Tang YL and Kim IM: Circular noncoding RNAs as potential therapies and circulating biomarkers for cardiovascular diseases. Acta Pharmacol Sin 39: 1100-1109, 2018.

15. von Harsdorf R, Li PF and Dietz R: Signaling pathways in reactive oxygen species-induced cardiomyocyte apoptosis. Circulation 99: 2934-2941, 1999.

16. Fu Y, Liu X, Zhang F, Jiang S, Liu J and Luo Y: Bortezomibinducible long non-coding RNA myocardial infarction associated transcript is an oncogene in multiple myeloma that suppresses miR-29b. Cell Death Dis 10: 319, 2019.

17. Yang K, Li W, Duan W, Jiang Y, Huang N, Li Y, Ren B and Sun J: Resveratrol attenuates pulmonary embolism associated cardiac injury by suppressing activation of the inflammasome via the MALAT1miR223p signaling pathway. Int J Mol Med 44: 2311-2320, 2019.

18. Kruger J and Rehmsmeier M: RNAhybrid: microRNA target prediction easy, fast and flexible. Nucleic Acids Res 34 (Web Server Issue): W451-W454, 2006.

19. Sticht C, De La Torre C, Parveen A and Gretz N: miRWalk: An online resource for prediction of microRNA binding sites. PLoS One 13: e206239, 2018.

20. Wang S, Ye L and Wang L: Protective mechanism of shenmai on myocardial ischemia-reperfusion through the energy metabolism pathway. Am J Transl Res 11: 4046-4062, 2019.

21. Pan JA, Tang Y, Yu JY, Zhang H, Zhang JF, Wang CQ and Gu J: miR-146a attenuates apoptosis and modulates autophagy by targeting TAF9b/P53 pathway in doxorubicin-induced cardiotoxicity. Cell Death Dis 10: 668, 2019.

22. Barwari T, Joshi A and Mayr M: MicroRNAs in cardiovascular disease. J Am Coll Cardiol 68: 2577-2584, 2016.

23. Romaine SP, Tomaszewski M, Condorelli G and Samani NJ: MicroRNAs in cardiovascular disease: An introduction for clinicians. Heart 101: 921-928, 2015. 
24. Schulte C, Karakas M and Zeller T: microRNAs in cardiovascular disease-clinical application. Clin Chem Lab Med 55: 687-704, 2017.

25. Wang K, Gan TY, Li N, Liu CY, Zhou LY, Gao JN, Chen C, Yan KW, Ponnusamy M, Zhang YH and Li PF: Circular RNA mediates cardiomyocyte death via miRNA-dependent upregulation of MTP18 expression. Cell Death Differ 24: 1111-1120, 2017

26. Aufiero S, Reckman YJ, Pinto YM and Creemers EE: Circular RNAs open a new chapter in cardiovascular biology. Nat Rev Cardiol 16: 503-515, 2019.

27. E S, Costa MC, Kurc S, Drożdż A, Cortez-Dias N and Enguita FJ: The circulating non-coding RNA landscape for biomarker research: Lessons and prospects from cardiovascular diseases. Acta Pharmacol Sin 39: 1085-1099, 2018.

28. Roush S and Slack FJ: The let-7 family of microRNAs. Trends Cell Biol 18: 505-516, 2008.

29. Balzeau J, Menezes MR, Cao S and Hagan JP: The LIN28/let-7 pathway in cancer. Front Genet 8: 31, 2017.

30. Bao MH, Feng X, Zhang YW, Lou XY, Cheng Y and Zhou HH: Let-7 in cardiovascular diseases, heart development and cardiovascular differentiation from stem cells. Int J Mol Sci 14: 23086-23102, 2013.

31. Zhou X, Sun F, Luo S, Zhao W, Yang T, Zhang G, Gao M, Lu R, Shu Y, Mu W, et al: Let-7a is an antihypertrophic regulator in the heart via targeting calmodulin. Int J Biol Sci 13: 22-31, 2017.

32. Seeger T, Xu QF, Muhly-Reinholz M, Fischer A, Kremp EM, Zeiher AM and Dimmeler S: Inhibition of let-7 augments the recruitment of epicardial cells and improves cardiac function after myocardial infarction. J Mol Cell Cardiol 94: 145-152, 2016.

33. Suarez Y, Fernandez-Hernando C, Pober JS and Sessa WC: Dicer dependent microRNAs regulate gene expression and functions in human endothelial cells. Circ Res 100: 1164-1173, 2007.
34. Cao L, Kong LP, Yu ZB, Han SP, Bai YF, Zhu J, Hu X, Zhu C, Zhu $S$ and Guo XR: microRNA expression profiling of the developing mouse heart. Int J Mol Med 30: 1095-1104, 2012.

35. Lederer M, Bley N, Schleifer C and Huttelmaier S: The role of the oncofetal IGF2 mRNA-binding protein 3 (IGF2BP3) in cancer. Semin Cancer Biol 29: 3-12, 2014.

36. Bhargava S, Patil V, Shah RA and Somasundaram K: IGF2 mRNA binding protein 3 (IMP3) mediated regulation of transcriptome and translatome in glioma cells. Cancer Biol Ther 19: 42-52, 2018.

37. Shimizu T, Sugihara E, Yamaguchi-Iwai S, Tamaki S, Koyama Y, Kamel W, Ueki A, Ishikawa T, Chiyoda T, Osuka S, et al: IGF2 preserves osteosarcoma cell survival by creating an autophagic state of dormancy that protects cells against chemotherapeutic stress. Cancer Res 74: 6531-6541, 2014.

38. Nishida K, Yamaguchi O and Otsu K: Crosstalk between autophagy and apoptosis in heart disease. Circ Res 103: 343-351, 2008.

39. Takemura G, Kanamori H, Okada H, Miyazaki N, Watanabe T, Tsujimoto A, Goto K, Maruyama R, Fujiwara T and Fujiwara H: Anti-apoptosis in nonmyocytes and pro-autophagy in cardiomyocytes: Two strategies against postinfarction heart failure through regulation of cell death/degeneration. Heart Fail Rev 23: 759-772, 2018.

This work is licensed under a Creative Commons Attribution-NonCommercial-NoDerivatives 4.0 International (CC BY-NC-ND 4.0) License. 